\title{
Suicide notes: Clinical and linguistic analysis from the perspective of the Interpersonal Theory of Suicide
}

\author{
Mercedes Fernández-Cabana ${ }^{a, *}$ \\ Francisco Ceballos-Espinoza ${ }^{b}$ \\ Raimundo Mateos ${ }^{a}$ \\ María Teresa Alves-Pérez ${ }^{c}$ \\ Alejandro Alberto García-Caballero ${ }^{a, d}$ \\ a Department of Psychiatry, School of \\ Medicine, Universidade de Santiago \\ de Compostela \\ b Department of Investigations Police \\ of Chile (PDI) \\ c Universidade de Vigo, NeCom \\ Research Group \\ d Complexo Hospitalario Universitario \\ de Ourense
}

SPAIN

CHILE

\begin{abstract}
Background and Objectives: Studying suicide notes is a strong methodology in suicidology and one of the most relevant theoretical frameworks is the Interpersonal Theory of Suicide proposed by Joiner.

This study analyzed 80 suicide notes collected in Chile searching for the risk factors proposed by the theory and its implications. The clinical and sociodemographic characteristics of the notes' authors were analyzed too.

Methods: The suicide notes were classified according to the presence or absence of the suicide risk factors proposed by the Interpersonal Theory of Suicide and were analyzed linguistically with the Linguistic Inquiry and Word Count program.

Results: One or both interpersonal risk factors indicated by the theory were identified in $60 \%$ of the notes. The "lack of belonging" factor was the most common risk factor, and various linguistic profiles in the suicide notes were found according to the presence or absence of this factor.

Conclusions: We confirmed the relevance of the interpersonal risk factors proposed as issues present in a high percentage of notes and found linguistic differences according to its presence or absence in the suicide notes.
\end{abstract}




\section{Introduction}

The World Health Organization states that each year an estimated 800,000 individuals die worldwide due to suicide ${ }^{1}$ and proposes the following suicide risk factors: mental or physical illness (especially chronic), drug/alcohol abuse, a history of violence, acute emotional stress, a significant and sudden change such as loss of employment, separation from a partner or other adverse event and, in many cases, a combination of these factors. It is accepted that suicidal behavior is influenced by a combination of biological, genetic, psychological, societal, contextual and situational factors.

Since the first studies by Shneidman ${ }^{2,3}$, there have been numerous studies on deaths by suicide and attempted suicides in an attempt to understand the phenomenon through an analysis of the notes that some individuals write before carrying out suicide ${ }^{4,5}$. The various theoretical approaches have emphasized the risk factors involved and have developed numerous theories that attempt to understand and prevent suicide. Our study approach consisted of the clinical and linguistic analysis of suicide notes using the theoretical framework of the Interpersonal Theory of Suicide (ITS) proposed by Thomas E. Joiner ${ }^{6}$.

ITS identifies 3 central constructs for explaining suicidal behavior. According to this model, the desire to end one's own life is related to the simultaneous presence of 2 interpersonal factors: the feeling of being a burden and lack of belonging to a group. The interaction of these 2 factors with a third factor, the acquired capability for suicide, puts the individual at risk of attempting suicide, with lethal or almost lethal results ${ }^{6}$.

In subsequent elaborations of the theory ${ }^{7}$ the feeling of being a burden includes 2 measures: a) the belief that one is so incapable or deficient as to represent a burden or responsi- bility for others and b) the emotionally charged cognition of hatred towards oneself. The indicators of these measures would be low self-esteem, feelings of guilt or shame and an agitated mental state. As indicated by Van Orden et al. (2010), individuals who feel they are a burden to the significant people in their life and who feel self-hatred are especially at risk. The presence of this factor is a predictor of suicide symptoms, previous suicide attempts and increased suicide risk, independent of other risk factors such as depressive symptoms, hopelessness and personality disorders. This factor occurs more often in elderly individuals, which is useful for understanding suicidal ideation in this population group ${ }^{8}$. Its interaction with the acquired capability to carry out suicide predicts an increase in suicide risk, beyond the influence of other factors such as depression, gender and age ${ }^{9}$. This factor correlates with low-quality social support from relatives and friends, as well as with mental and physical symptoms ${ }^{10}$. A review of the available evidence on the relevance of the "feeling like a burden" factor found support for the association between this factor and suicidal ideation and tested its usefulness for increasing the predictability of ideation once other risk factors had been controlled. In short, the results of the previous research show a correlation between this factor and a history of attempted suicide and support its relationship with death by suicide ${ }^{11}$.

The second interpersonal factor, a lack of belonging, is a multidimensional construct that includes not only the feeling of loneliness but also the lack of reciprocal care from the community. This factor involves a dynamic cognitive-affective condition whose most severe form involves the perception that there are no significant and mutual support relationships and whose chronicity increases this perception ${ }^{7}$. This factor has been linked to a history of attempted suicide in individu- 
als undergoing treatment for opioid-dependence $^{12}$ and addictions in general. The lower level of perceived social support has also been related to attempted suicide ${ }^{13}$. The combined presence of the "feeling like a burden" and "lack of belonging" factors significantly correlates with suicidal ideation ${ }^{9,10}$, and their interaction provides additional variance to explain this ideation, especially in young individuals for whom "lack of belonging" is a better predictor ${ }^{14}$.

Lastly, the theory indicates that the capability to carry out the suicide could be acquired through habituation to the fear and pain involved in self-harm and the strengthening of opposing processes in response to this fear and pain. The opponent-process theory ${ }^{15}$ states that the initial fear response when faced with a painful or fear-inducing experience will decrease with repeated trials, while the subsequent response of relief or exhilaration will be strengthened with repeated exposure to these experiences (attempted suicide, family history of suicide, childhood abuse, combat experiences, etc.). The acquired capability for suicide includes 2 measures: reducing the fear of death and increasing tolerance to physical pain, which would lead to the assessment that the pain involved in carrying out the chosen method of suicide would be tolerable ${ }^{7}$. This factor, measured with a scale, is greater in males and in individuals with previous suicide attempts and/or a history of provocative or painful experiences ${ }^{9}$. An individual's acquired capability for suicide is determined from their history of attempted suicide ${ }^{16}$, self-harm or being witness to the injuries or pain of others, although the construct requires greater specification ${ }^{17}$. The increase in this capability by repeated exposure to death and pain could be behind the high number of suicides among soldiers who return to society after experiencing combat ${ }^{18}$. It has been hypothesized that impulsivity increases this capability, although studies have shown an indirect influence through increased provocative and painful life experiences in individuals with this characteristic ${ }^{19}$.

Using the ITS as a reference framework, there has been a search for risk factor indicators in the texts and suicide notes, using automated linguistic analysis of these writings and their qualitative assessment.

In terms of the linguistic analysis, the Linguistic Inquiry and Word Count program (LIWC) $)^{20}$ analyzes written text word by word and calculates the percentage of words that meet each of the 72 categories of the text's language. In recent years, numerous studies have been conducted using the LIWC program. Several of the program categories have been linked to various psychological processes ${ }^{21}$, including the risk of suicide ${ }^{22-26}$. Williams (2006) analyzed musician Kurt Cobain's diary and suicide note, as well as letters from another man who died by suicide, comparing them with texts written by nonsuicidal individuals ${ }^{27}$. Williams found an increased use of words from the language categories "religion", "certainty" and "optimism" as the moment of death approached (the use of these categories did not increase in the controls) and interpreted these results as the fruit of a possible increase in thoughts on the religious or spiritual implications of suicide, as well as the feelings of competence and conviction for carrying out the act. Williams also investigated whether there were different language patterns in the writings that expressed "feeling like a burden" and/or "lack of belonging" (in individuals with no history of suicidal behavior). The author found that "lack of belonging" correlated positively with positive emotion indices and negatively with negative emotion indices in these writings (analyzed with LIWC), while "feeling like a burden" correlated positively with both. 
From a qualitative approach, suicide notes have been analyzed in an attempt to detect signs of the interpersonal factors of ITS ${ }^{6}$. Attempted suicide notes have been compared with notes from individuals who died by suicide. It was found that "feeling like a burden" correlated only with consummated suicide and with the selection of suicide methods of greatest lethality ${ }^{28}$. The conclusion was that the perception of feeling like a burden for loved ones would be a characteristic of suicide. Other studies, however, have had conflicting results. Gunn et al., in a qualitative analysis of 261 notes, indicated that the "lack of belonging" factor was present in $30.7 \%$ of the notes (with a higher frequency in the young than in the elderly). The "feeling like a burden" factor was present in $10.3 \%$ (not age-related but genderrelated, with a greater frequency in suicide notes written by females). These 2 factors showed up in only 11 of the analyzed notes $(4.2 \%)^{29}$. Moreover, another analysis of 664 suicide and attempted suicide notes (which included 33 simulated notes) showed the presence of the "lack of belonging" factor in $42.5 \%$ of the notes. The "feeling like a burden" factor occurred in $15.5 \%$ of the notes, and both factors were in $9.5 \%$ of the analyzed notes ${ }^{30}$.

Most of the studies mentioned above were conducted with English suicide notes or texts; none of the studies analyzed Spanish suicide notes. The only LIWC analysis we know of in Spanish was conducted with a small number of suicide notes collected in Spain, which found language differences depending on the authors' sex, age and community ${ }^{31}$. However, as far as we know, Spanish suicide notes have not been qualitatively analyzed in search of indicators of risk factors proposed by the ITS. The LIWC has also not been applied to detect specific language profiles according to the presence or absence of these factors.

We therefore established the following objectives and hypotheses (based on previous studies):
- Analyze whether there is a relationship between the authors' sex or age and other recorded clinical and demographic characteristics. We hypothesized that such a relationship exists and that we would find differences by sex and age in suicide methods used and triggers of suicide.

- Qualitatively analyze the suicide notes for content that refers to the interpersonal risk factors of ITS and determine in what measure these references appear in the 80 notes collected in Chile, adding the third factor proposed by the ITS: "acquired capability for suicide". Our hypothesis was that we would find the "lack of belonging" factor more often and that these factors would be present in a high percentage of the analyzed notes.

- Ascertain whether there is a relationship between these factors and variables in the authors of suicide notes, such as sex, age and living with a partner at the time of the death. Our hypothesis was that the "lack of belonging" factor would be more common in young individuals, the "perceived burdensomeness" factor would be more common in elderly people and in women, and men would show a higher "acquired capability for suicide".

- Classify the cases by the relative lethality of the suicide method and study whether there is a relationship between this variable and ITS risk factors and/or other characteristics of the notes' authors. Our hypothesis was that the "perceived burdensomeness" and the "acquired capability to suicide" factors would be related with higher lethality, and that such lethality would be higher in men and elderly people.

- Analyze the suicide notes with the Spanish version of the LIWC program ${ }^{32}$ to ascertain whether there are differentiated language profiles according to the sex or age 
of the notes' authors or between the notes that refer to "lack of belonging" and those that refers to "feeling like a burden". Our hypothesis was that we would find such different language profiles and that the percentage of use of positive and negative emotion words would be different in notes which include content related to these factors and those without it.

\section{Method}

\section{Sample of suicide notes}

The sample of suicide notes was collected based on a review of official records of deaths registered as suicides in Chile from January 2010 to June 2012 and investigated by the Homicide Brigade of the Chilean Investigative Police. To this end, their case files for death by suicide were reviewed individually, until a total of 80 suicide nodes were found in the records ${ }^{\S}$.

The total sample of individuals who died by suicide $(\mathrm{n}=2,272)$ included individuals between 8 and 94 years of age. A total of 1,877 $(82.61 \%)$ were male, $1,311(57.70 \%)$ were single or similar and the most common mechanism for death was hanging $(51.76 \%, \mathrm{n}=1,174)$, followed by firearms $(9.68 \%, \mathrm{n}=220)$ and poisoning $(4.80 \%, \mathrm{n}=109)$.

\section{Procedure}

The individuals whose report contained a suicide note were characterized by their demographic and forensic characteristics. These included the date of suicide, age, sex, residen- ce, marital status, educational level, employment status, method of death, number of suicide notes, history of psychiatric diagnosis or treatment and previous attempts (not necessarily registered in the report), depressive symptoms (with no medical diagnosis or treatment) and supposed triggers (reported by individuals in their community in response to a semistructured interview and based on what was written in the notes and/or their knowledge about the deceased), analyzing whether there was a relationship between the author's sex or age and other previously mentioned characteristics. The individuals were also classified according to the lethality of the method chosen for carrying out suicide, following the criteria proposed in previous studies ${ }^{28}$. Methods that resorted to poisoning, overdosing, asphyxiation or knives were classified as "relatively low lethality", while methods that included hanging, jumping and firearms were classified as "relatively high lethality".

All personal data were processed according to the directives of the World Health Organization and the Declaration of Helsinki.

To search for ITS factors, each note was classified independently by 3 judges (a clinical psychologist, a forensic psychologist and a psychiatrist, all authors of this study) according to whether the contents referred to "feeling like a burden" and "lack of belonging" factors. To this end, we followed the criteria proposed in previous studies ${ }^{29,30}$ where the presence of perceived burdensomeness was related to any reference to being a burden (emotional, financial...) on those around, references to others being "better off" with their death and feeling that their deaths would bring about something good. Criteria for thwarted belongingness included feelings of being isolated or discon-

\footnotetext{
$\S$ Given that Chile does not have a registry of suicide notes, and these are not always included in the report, the number of cases with suicide notes cannot be construed as an actual percentage of the total number of suicides.
} 
nected from others, feelings as though they do not fit in or feeling lonely (and concern about it) and suicide related to a recent loss. Each suicide note was scored for both factors, indicating whether each was "clearly present", "possibly present" or "absent". For the purposes of this study, we combined the "clearly present" and "possibly present" results.

The third factor proposed by the ITS ("an acquired capability for suicide") was classified as present in those individuals for whom we had proof of at least one previous suicide attempt or a history of suicide in the family of origin following previous criteria ${ }^{16}$. We analyzed the potential relationships between the 3 ITS factors and the relative lethality of the chosen method and between these factors and other characteristics of the suicide note authors.

For the linguistic analysis, we analyzed the written material collected in each case as a continuous text using the Spanish version of the LIWC $^{32}$. We linked their results to their authors' gender and age, as well as to the presence or absence of the interpersonal factors of suicide risk established in the ITS $^{6}$.

\section{Statistical analysis}

We performed a descriptive analysis of the data. To compare categorical variables, we employed the chi-squared test. KolmogorovSmirnov normality tests were also performed. For non-Gaussian variables, we employed the nonparametric Mann-Whitney $U$ and Kruskal-Wallis tests. Using the single-factor ANOVA test, we studied the relationship of the various LIWC categories with age at death. 3 age groups were established after performing a GAM (Generalized Additive Model) using smoothing p-spline, which showed the nonlinear character of this variable, observing three separate areas which were grouped in 3 ranges: 0-30 years, 31-60 years and 61-90 years. Bonferroni correction was made to the control of the Type I error. To calculate the agreement among the judges in the classification of notes according to the presence/absence of ITS factors, we employed the Kappa index.

We considered p-values $<0.05$ as statistically significant and the analysis were performed with SPSS 15.0.

\section{Results}

\section{Clinical and demographic characteristics; relationship with sex and age of the deceased}

The characteristics of the sample of individuals who left a suicide note are listed in the following table (see Table 1).

We found no significant differences by sex in terms of having a partner or not at the time of the death $(p=0.279)$, living in a rural or urban area $(p=0.931)$, the number of suicide notes written (mean of 2 for males and 2.4 for females; $p=0.564$ ) or the age at the time of death (mean age for males, 47.18 years [SD, 19.36]; mean age for females, 39.79 [SD, 14.76]; $p=0.099$ ). There were significant differences in the chosen method of suicide, with a greater use of hanging in males $(p=0.001)$ and poisoning in females $(p=0.025)$. More females $(n=10 ; 41.67 \%$ of the deceased) than males $(n=6 ; 10.71 \%)$ had a history of psychiatric problems $(p=0.002)$. The presence of mental illness was reported as the trigger in more females than males $(p=0.038)$ (see Table 2).

We found no significant differences by age in terms of whether the victim had a partner or not at the time of death $(p=0.711)$ nor did we find a relationship between age and urban/rural setting $(p=0.970)$ or method of sui- 
Table 1

Characteristics of the sample of individuals who left a suicide note.

Sample characteristics

\begin{tabular}{|c|c|}
\hline \multicolumn{2}{|l|}{ Sex and age } \\
\hline Male & $56(70 \%)$ \\
\hline Females & $24(30 \%)$ \\
\hline Mean age (SD) & $45(\mathrm{SD}, 18.33)$ \\
\hline Age range & $14-90$ years \\
\hline 0-30 years & $19(23.75 \%)$ \\
\hline $31-60$ years & $46(57.5 \%)$ \\
\hline $61-90$ years & $15(18.75 \%)$ \\
\hline \multicolumn{2}{|l|}{ Setting } \\
\hline Urban & $73(91.25 \%)$ \\
\hline Rural & $7(8.8 \%)$ \\
\hline \multicolumn{2}{|l|}{ Marital status } \\
\hline Married or with partner & $53(66.3 \%)$ \\
\hline Others & $27(33.75 \%)$ \\
\hline \multicolumn{2}{|l|}{ Suicide method } \\
\hline Hanging & $58(72.5 \%)$ \\
\hline Firearm & $6(7.5 \%)$ \\
\hline Poisoning & $5(6.25 \%)$ \\
\hline Jumping & $4(5 \%)$ \\
\hline Knife & $3(3.75 \%)$ \\
\hline Monoxide inhalation & $2(2.5 \%)$ \\
\hline Vehicular impact & $1(1.25 \%)$ \\
\hline Electrocution & $1(1.25 \%)$ \\
\hline \multicolumn{2}{|l|}{ Suicide trigger } \\
\hline Emotional problems & $36(45 \%)$ \\
\hline Financial or legal problems & $20(25 \%)$ \\
\hline Physical disease & $11(13.75 \%)$ \\
\hline Mental illness & $7(8.75 \%)$ \\
\hline Unknown & $6(7.5 \%)$ \\
\hline \multicolumn{2}{|l|}{ Psychiatric/treatment history } \\
\hline Present & $16(20 \%)$ \\
\hline \multicolumn{2}{|l|}{ Untreated depressive symptoms } \\
\hline Present & $31(38.75 \%)$ \\
\hline \multicolumn{2}{|l|}{ Number of notes } \\
\hline 1 & $49(61.25 \%)$ \\
\hline 2 or more & $31(38.75 \%)$ \\
\hline
\end{tabular}


Table 2

Differences by sex in the supposed trigger, history of mental illness and method of suicide used.

\begin{tabular}{lrrr} 
& Male & Female & $p^{*}$ \\
\hline Trigger & & & \\
Emotional problems & 23 & 13 & 0.405 \\
Financial/legal problems & 15 & 5 & 0.778 \\
Physical disease & 10 & 1 & 0.202 \\
Unknown & 6 & 0 & 0.229 \\
Mental illness & 2 & 5 & $\mathbf{0 . 0 3 8}$ \\
\hline Mental illness history & & & \\
Psychiatric history & 6 & 10 & $\mathbf{0 . 0 0 2}$ \\
Untreated depressive symptoms & 23 & 8 & 0.515 \\
Total & 29 & 18 & 0.053 \\
\hline Suicide method & & & \\
Hanging & 47 & 11 & $\mathbf{0 . 0 0 1}$ \\
Firearm & 5 & 5 & 0.781 \\
Poisoning & 0 & 3 & $\mathbf{0 . 0 2 5}$ \\
Jumping & 1 & 2 & 0.146 \\
Knife & 1 & 0 & 0.441 \\
Monoxide inhalation & 2 & 1 & 0.876 \\
Electrocution & 0 & 1 & 0.661 \\
Vehicular impact & 0 & & 0.661 \\
\hline
\end{tabular}

p*: chi-squared test.

cide employed ( $p=0.353)$. In terms of supposed suicide trigger, the individuals with physical disease had a statistically higher mean age (64.91 years; SD, 19.27 years; $p=0.002$ ) than the individuals with emotional, financial or legal problems (42 years), those with mental illness (41 years) and those with unknown triggers (38 years).

\section{Tracking of Interpersonal Theory of Suicide factors}

In the analysis of ITS characteristics, initially the 3 judges completely agreed on the classification of 62 of the 80 suicide notes (77.5\%), with an agreed upon Kappa index between 0.8 and 0.92 in that initial classification, after which we met to resolve the dis- crepancies. In 48 of the suicide notes reviewed $(60 \%)$, we found content related to the interpersonal risk factors of ITS. The "lack of belonging" factor was the most common and was detected in $42.5 \%$ of the notes $(n=34)$, while the "feeling like a burden" factor was scored in $35 \%$ of the notes $(n=28)$. We found content referring to both risk factors in 14 of these notes. The third factor or "acquired capability for suicide" was scored as present in 14 cases $(17.5 \%)$.

We analyzed the relationships between the 3 ITS factors and sex, age and living with a partner or not at the time of the death. Only the relationship between "acquired capability" and sex $(p=0.015)$ was significant. This factor was present in more females (8 of 24; $33.3 \%$ ) than males ( 6 of $56 ; 10.7 \%)$. 


\section{Relative lethality of the suicide method and its relationship with other variables}

Of the 80 cases analyzed, $70(87.5 \%)$ were classified as suicides with a relatively high lethality method, while the remaining 10 $(12.5 \%)$ were classified as relatively low lethality. The analysis of the 2-to-2 relationship between "relative lethality of the chosen method" and "acquired capability", "lack of belonging" or "feeling like a burden" factors did not achieve statistical significance. The 3 ITS factors analyzed jointly also were not predictors of greater relative lethality of the suicide method. In the analysis of the relationship between relative lethality and sex, supposed trigger, age or living with a partner or not at the time of suicide, only the relationship between lethality and sex $(p=0.003)$ was significant. More males chose relatively high lethality methods (53 of $56 ; 94.6 \%$ ) than females ( 17 of $24 ; 70.8 \%$ ).

\section{Linguistic analysis of the suicide notes}

When comparing the usage of words included in the linguistic categories of the LIWC according to sex, we found significant differences ( $p=0.002)$ only in the use of words in the "human" category, which was higher in females (mean, 2.61; SD, 2.60) than in males (mean, 1.13; SD, 1.06).

Taking into account the victim's age, the younger individuals used more affective words $(p=0.001)$, specifically words about positive emotion $(p=0.004)$ and feelings $(p=0.001)$, as well as more words about "cognitive mechanisms" ( $p=0.041)$ and references to the present $(p=0.026)$. The older individuals used more articles $(p=0.015)$, punctuation marks $(p=0.011)$ and longer words $(p=0.007)$; made more references to occupations $(p=0.001)$ and money $(p=0.007)$; and had a lower usage of words in the "social" category ( $p=0.019)$ (see Table 3 ).

Table 3

LIWC categories with significant differences in the usage rate according to the victim's age.

\begin{tabular}{lcccc} 
LIWC Categories-Age & $\begin{array}{c}0-30 \\
\text { Mean (SD) }\end{array}$ & $\begin{array}{c}31-60 \\
\text { Mean }(\mathrm{SD})\end{array}$ & $\begin{array}{c}61-30 \\
\text { Mean }(\mathrm{SD})\end{array}$ & $P^{*}$ \\
\hline Affective & $11.57(5.63)$ & $8.70(3.83)$ & $6.09(2.73)$ & 0.001 \\
- Positive emotion & $8.26(4.1)$ & $5.73(4.14)$ & $3.73(2.37)$ & 0.004 \\
- Positive feelings & $6.30(4.19)$ & $4.11(3.83)$ & $1.58(1.10)$ & 0.001 \\
Cognitive mechan. & $10.92(3.66)$ & $8.56(3.70)$ & $8.29(3.10)$ & 0.041 \\
Present & $11.24(3.13)$ & $9.27(3.98)$ & $7.82(3.17)$ & 0.026 \\
Articles & $6.69(7.86)$ & $5.53(2.79)$ & $9.58(3.37)$ & 0.015 \\
Punctuation marks & $8.38(6.02)$ & $9.26(5.66)$ & $14.93(10.01)$ & 0.011 \\
Words $>$ 6 letters & $13.52(5.78)$ & $15.86(6.47)$ & $20.35(5.52)$ & 0.007 \\
Occupation & $0.82(0.79)$ & $1.10(0.95)$ & $2.21(1.63)$ & 0.001 \\
Money & $0.25(0.49)$ & $0.27(0.50)$ & $0.79(0.77)$ & 0.007 \\
Social & $15.61(3.83)$ & $15.75(7.70)$ & $10.47(3.10)$ & 0.019 \\
\hline
\end{tabular}

*: single-factor ANOVA test. 
When comparing the usage of words included in the linguistic categories of the LIWC according to the presence or absence in the suicide note of the "lack of belonging" factor, we found significant differences in the use of words in the " 1 st person plural" $(p=0.002)$,
"2 $2^{\text {nd }}$ person" ( $\left.p=0.027\right)$, "negative emotions" ( $p=0.001)$ and "human" $(p=0.038)$ categories. This usage was higher in the notes in which this factor was identified; these notes were also significantly longer $(p=0.000)$ (see Table 4).

Table 4

LIWC categories with significant differences in the usage rate according to the presence/absence of the "lack of belonging" factor.

\begin{tabular}{lrrr} 
LIWC Categories - Lack of belonging & \multicolumn{1}{c}{$\begin{array}{c}\text { Present } \\
\text { Mean (SD) } \\
\text { Median (min-max) }\end{array}$} & $\begin{array}{c}\text { Absent } \\
\text { Mean (SD) } \\
\text { Median (min-max) }\end{array}$ & \multicolumn{1}{c}{$P^{*}$} \\
\hline Word count & $\begin{array}{c}\text { 491.09 (538.08) } \\
237.50(30-2232)\end{array}$ & $99(2-2812)$ & 0.000 \\
\hline $1^{\text {st }}$ person plural & $0.36(0.51)$ & $0.12(0.34)$ & 0.002 \\
\hline $2^{\text {nd }}$ person & $0.18(0-1.96)$ & $0(0-2.04)$ & \\
\hline Negative emotions & $1.81(1.67)$ & $1.17(1.48)$ & 0.027 \\
\hline Humans & $1.69(0-8.10)$ & $0.38(0-5.03)$ & \\
\hline
\end{tabular}

*: Mann-Whitney U test.

When comparing the usage of words included in the linguistic categories of the LIWC according to the presence or absence in the suicide note of the "feeling like a burden" factor, we found significant differences in the use of words in the "optimism" $(p=0.012)$, "cause" $(p=0.002)$ and "achievement" $(p=0.033)$ categories. This usage was greater in the notes with this factor. In this case, the notes were also longer than those without this factor $(p=0.001)$ (see Table 5).

\section{Discussion}

The most often chosen method of suicide according to sex (hanging in males and poisoning in females) was similar to that found in previous European studies ${ }^{33}$.

The presence of mental illness is a significant risk factor for suicide. It is estimated that a high percentage of individuals who die from suicide have this condition ${ }^{34,35}$. Previ- 
Table 5

LIWC categories with significant differences in the usage rate according to the presence/absence of the "feeling like a burden" factor.

\begin{tabular}{|c|c|c|c|}
\hline LIWC Categories - Feeling like a burden & $\begin{array}{c}\text { Present } \\
\text { Mean (SD) } \\
\text { Median (min-max) }\end{array}$ & $\begin{array}{c}\text { Absent } \\
\text { Mean (SD) } \\
\text { Median (min-max) }\end{array}$ & $P^{*}$ \\
\hline \multirow[t]{2}{*}{ Word count } & $462.11(599.11)$ & $255.88(410.09)$ & \multirow{2}{*}{0.001} \\
\hline & $335.50(13-2812)$ & $95(2-2232)$ & \\
\hline \multirow[t]{2}{*}{ Optimism } & $0.67(0.52)$ & $0.46(0.80)$ & \multirow{2}{*}{0.012} \\
\hline & $0.64(0-2)$ & $0(0-5)$ & \\
\hline \multirow[t]{2}{*}{ Cause } & $0.81(0.55)$ & $0.51(0.82)$ & \multirow{2}{*}{0.002} \\
\hline & $0.74(0-1.85)$ & $0(0-4)$ & \\
\hline \multirow[t]{2}{*}{ Achievement } & $1.11(0.77)$ & $0.80(1.05)$ & \multirow{2}{*}{0.033} \\
\hline & $1.07(0-3.19)$ & $0.35(0-4.65)$ & \\
\hline
\end{tabular}

*: Mann-Whitney U test.

ous studies have shown that suicidal ideation is related to the presence of depression and that planned and attempted suicide is related to disorders characterized by anxiety or agitation and poor impulse control ${ }^{36}$. In our sample, the relatives identified the presence of mental illness as the supposed trigger in only 7 cases and more often in females, possibly because we have more females with previous suicide attempts, which makes the presence of mental health problems more visible. Assuming that a trigger may be on the mind of the victim without being displayed in its suicide note or known by their relatives, is striking the limited election of mental illness as the trigger, given that there was a history of psychiatric treatment or untreated depressive symptoms in 47 of the deceased. This fact could be related to the stigma that surrounds mental illness ${ }^{37}$ and the burden and stigma by association that this frequently represents for the family ${ }^{38}$. Of these 47 cases, 18 were female (of 24 deceased), with more females than males undergoing psychiatric treatment (41.67\% vs. $10.71 \%$, respectively) which agrees with the already reported greater willingness by females to seek help ${ }^{39}$. Only 16 of the deceased $(20 \%)$ had a history of psychiatric problems, which brings us to the low level of effort in searching for mental health treatment by individuals at risk for suicide. International studies have linked this problem with the low perceived need for treatment and the negative attitudes towards the search for this treatment (the desire to handle it without help, the belief that it will improve on its own, that it is not a serious problem or that treatment will not be effective, the associated stigma, etc.). Studies have also linked this problem to structural barriers such as difficulties accessing treatment ${ }^{40}$.

The individuals for whom physical disease was considered the suicide trigger were older, an expected result given the increased morbidity associated with aging, which constitutes a risk factor for suicide per $s e^{1}$ and given the presence of a positive correlation between suicide and dependence in elderly individuals ${ }^{41}$. 
In terms of the qualitative analysis of the suicide notes, we found content referring to the ITS risk factors of "lack of belonging" and "feeling like a burden" at higher rates than in the study by Gunn et al. (the only study that analyzed suicide notes exclusively $)^{29}$. One or both of these factors appeared in $60 \%$ of the analyzed notes. The "lack of belonging" factor was more common in previous studies $^{29,30}$ and was in tune with the fact that emotional problems were the more frequently indicated trigger.

In general, it is commonly accepted that females attempt suicide more often than ma$\mathrm{les}^{42}$. It has been hypothesized that it is more common for females to attempt suicide as a means of communicating their discomfort or to achieve changes in their environment (suggesting a lower suicidal intent). This hypothesis is controversial, however, given that the degree of intent does not necessarily correlate with the lethality of the chosen method ${ }^{43}$, and the result could be determined by the selection of the method ${ }^{44}$. The higher rate of attempted suicide in females could explain why the ITS "acquired capability" factor was more common in our study in the cases where the deceased was a female, given that the presence of previous suicide attempts was one of the criteria we used to identify this factor. This result does not agree with those of earlier studies, possibly because in those studies this capability was estimated by taking more variables into account ${ }^{10}$. We also found no correlation between content referring to the "feeling like a burden" factor and older age ${ }^{8}$, possibly due to the scarcity of individuals in our sample older than 61 years $(18.75 \%)$. We found no relationship between the "lack of belonging" factor and youth in the notes ${ }^{29}$ or between this factor and living with a partner or not at the time of death.

In contrast to previous findings ${ }^{28}$, there was no correlation in our sample between the relative lethality of the chosen method and other ITS factors, although this result may be influenced by the shortage of people with relatively low lethality methods of suicide in our sample. We confirmed the selection of more lethal suicide methods among males than in females ${ }^{45}$.

Lastly, in the linguistic analysis of the notes, the higher usage of words from the LIWC category "human" in the notes written by females could indicate (as noted by Newman $e t$ al.) that females have a greater interest in communicating psychological and societal issues ${ }^{46}$, as well as reflect the greater influence of interpersonal problems on females as a risk factor of suicide ${ }^{47}$.

The language used in the suicide notes of the youngest individuals showed a higher rate of affective and positive emotion words. The use of emotion words would be an indicator of greater personal immersion in the writing, and the use of the present time provides closeness and warmth ${ }^{48,49}$. The greater use of words from the "cognitive mechanisms" category would indicate greater reflection and focus on the underlying meaning of the topic ${ }^{50}$. The language used by the elderly in their suicide notes is, in contrast, more elaborate and careful (with a higher usage of grammatical particles and long words) and is more focused on specific issues (money, occupation) and with fewer indicators of social connection and integration ${ }^{51}$.

These results do not agree with the findings from the analysis of suicide notes from Spain, in which the notes written by females were longer and used a greater percentage of words related to feelings and emotions, more verbs in the past and future and more first-person plural pronouns than those written by males. The notes of younger individuals were different from those written by older individuals only in the greater use of punctuation marks ${ }^{31}$. Given that Spanish is spoken by more than 500 million individuals worldwide who live in 
various countries and cultures, we must consider the linguistic differences between South American Spanish (which is neater and more nuanced, with longer greetings and farewells) and the Spanish spoken in Spain (which is shorter and more specific, among other differences). These differences are accentuated in the popular language ${ }^{52}$ and could explain the divergence in the results.

We found differences in the language used in the notes according to the presence or absence of content related to ITS factors, but not uniquely regarding use of emotion words. The suicide notes that included these factors were longer; the notes in which the "lack of belonging" factor was detected made greater use of the second-person singular pronoun and the first-person plural pronoun, as well as words in the "human" and "negative emotions" categories (words such as abandonment, grief and isolation). These notes therefore referred more frequently to difficulties with the reference group and with the important individuals for the authors of the notes.

The suicide notes that included references to the "feeling like a burden" factor included a greater percentage of words of the "cause", "optimism" and "achievement" categories. These notes are more focused on explaining the reasons for the decision and emphasizing the benefits for those left behind, who would be "better off once the individual has gone away". These results were different from those found previously ${ }^{27}$ although in our sample we analyzed suicide notes and not texts written by individuals without a history of suicide.

\section{Conclusions}

This study presents the clinical and demographic characteristics of the study sample, with only $20 \%$ of the deceased having a his- tory of mental health treatment. More females than males were undergoing treatment. The use of poisoning as a suicide method was more common among females, while males preferred the use of hanging, choosing suicide methods with relatively greater lethality. The families most often indicated mental illness as the trigger in females and physical disease for elderly individuals.

We confirmed the presence of contents related to the interpersonal risk factors proposed in Joiner's ITS in a significant percentage $(60 \%)$ of suicide notes, as well as a greater frequency of the "lack of belonging" factor, possibly related to the most common supposed trigger: emotional problems. Therefore, these factors should be considered and assessed in clinical settings to improve understanding and, above all, prevention of suicide.

The linguistic analysis found differences according to the authors' gender and age and revealed the need to consider the cultural differences in its interpretation. We also found linguistic differences according to the presence or absence of ITS factors, which supports the construct validity of these factors that could be tracked on Internet forums and blogs. As far as we know, our study is the first to analyze suicide notes in Spanish from this theoretical perspective.

As for study limitations, there is the use of the qualitative methodology for classifying suicide notes according to the presence or absence of ITS factors, which might be subject to biases. In addition, there was the decision to follow the criteria proposed by previous studies to decide the acquired capability and relative lethality of the suicide method for each case, criteria that consider a limited number of variables in making this classification. The design of our study would be stronger if we had a sample of notes written by attempters to compare with as this will allow us to distin- 
guish patterns that indicate increased risk among people who carry out suicidal behaviors. Another limitation is due to the characteristics of the linguistic analysis tools, given that LIWC performs a quantitative analysis that does not allow for contextual influence or double meanings in the text. Its classification of words is based on probabilistic models of language use and therefore is more reliable to greater number of analyzed words.

\section{References}

1. WHO. Public Health Action for the Prevention of Suicide: A framework. Geneva: Department of Mental Health and Substance Abuse: World Health Organization; 2012 [cited 201426 jun]. Disponible en: http://www.who.int/mental_health/prevention/suicide/suicideprevent/en/].

2. Shneidman ES, Farberow NL. Clues to suicide. Public Health Reports. 1956; 71(2): 109-14.

3. Shneidman ES. Suicide, lethality, and the psychological autopsy. International Psychiatry Clinics. 1969; 6(2): 225-50.

4. Shneidman ES. Comprehending suicide. Landmarks in 20th-century suicidology. Washington, DC: American Psychological Association; 2001.

5. Shneidman ES. Autopsy of a suicidal mind. New York: Oxford University Press; 2004.

6. Joiner TE. Why people die by suicide. Cambridge, MA: Harvard University Press; 2005.

7. Van Orden KA, Witte TK, Cukrowicz KC, Braithwaite SR, Selby EA, Joiner TE. The interpersonal theory of suicide. Psychological Review. 2010; 117(2): 575-600.

8. Van Orden KA, Lynam ME, Hollar D, Joiner TE. Peirceived burdensomeness as an indicator of suicidal symptoms. Cognitive Therapy and Research. 2006; 30: 457-67.

9. Van Orden KA, Witte TK, Gordon KH, Bender TW, Joiner TE. Suicidal desire and the capability for suicide: tests of the interpersonal-psychological theory of suicidal behavior among adults. Journal of Consulting and Clinical Psychology. 2008; 76(1): 72-83.

10. Christensen H, Batterham PJ, Mackinnon AJ, Donker T, Soubelet A. Predictors of the risk factors for suicide identified by the interpersonal-psychological theory of suicidal behaviour. Psychiatry Research. 2014; 219(2): 290-7.
11. Hill RM, Pettit JW. Perceived burdensomeness and suicide-related behaviors in clinical samples: current evidence and future directions. Journal of Clinical Psychology. 2014; 70(7): 631-43.

12. Conner KR, Britton PC, Sworts LM, Joiner TE. Suicide attempts among individuals with opiate dependence: the critical role of belonging. Addictive Behaviors. 2007; 32(7): 1395-404.

13. You S, Van Orden KA, Conner KR. Social connections and suicidal thoughts and behavior. Psychology of Addictive Behaviors: Journal of the Society of Psychologists in Addictive Behaviors. 2011; 25(1): 180-4.

14. Christensen H, Batterham PJ, Soubelet A, Mackinnon AJ. A test of the Interpersonal Theory of Suicide in a large community-based cohort. Journal of Affective Disorders. 2013; 144(3): 225-34.

15. Solomon RL, Corbit JD. An opponent-process theory of motivation. I. Temporal dynamics of affect. Psychological Review. 1974; 81(2): 119-45.

16. Joiner TE, Van Orden KA, Witte TK, Selby EA, Ribeiro JD, Lewis R, et al. Main predictions of the interpersonal-psychological theory of suicidal behavior: empirical tests in two samples of young adults. Journal of Abnormal Psychology. 2009; 118(3): 634-46.

17. Ribeiro JD, Joiner TE. The interpersonal-psychological theory of suicidal behavior: current status and future directions. Journal of Clinical Psychology. 2009; 65(12): 1291-9.

18. Selby EA, Anestis MD, Bender TW, Ribeiro JD, Nock MK, Rudd MD, et al. Overcoming the fear of lethal injury: evaluating suicidal behavior in the military through the lens of the Interpersonal-Psychological Theory of Suicide. Clinical Psychology Review. 2010; 30(3): 298-307.

19. Bender TW, Gordon KH, Bresin K, Joiner TE. Impulsivity and suicidality: the mediating role of painful and provocative experiences. Journal of Affective Disorders. 2011; 129(1-3): 301-7.

20. Pennebaker JW, Francis ME, Booth RJ. Linguistic Inquiry and World Count (LIWC): LIWC2001. Mahwah, NJ: Erlbaum; 2001.

21. Tausczik YR, Pennebaker JW. The psychological meaning of words: LIWC and computerized text analysis methods. Journal of Language and Social Psychology. 2010; 29(1): 24-54.

22. Stirman SW, Pennebaker JW. Word use in the poetry of suicidal and nonsuicidal poets. Psychosomatic Medicine. 2001; 63(4): 517-22.

23. Pennebaker JW, Stone LD. What was she trying to say? In: Lester D, editor. Katie's diary: Unlocking the mistery of a suicide. New York: Brunner-Routledge; 2004. p. 55-79. 
24. Barnes DH, Lawal-Solarin FW, Lester D. Letters from a suicide. Death Studies. 2007; 31(7): 671-8.

25. Lester D, Haines J, Williams CL. Content differences in suicide notes by sex, age, and method: a study of Australian suicide notes. Psychological Reports. 2010; 106(2): 475-6.

26. Handelman LD, Lester D. The content of suicide notes from attempters and completers. Crisis. 2007; 28(2): $102-4$.

27. Williams FM. The relationship among linguistic patterns, twarted belongingness, perceived burdensomeness, and suicidal behavior: A test of Joiner s theory of suicide. Electronic Theses, Treatises and Dissertations 2006.

28. Joiner TE, Pettit JW, Walker RL, Voelz ZR, Cruz J, Rudd MD, et al. Perceived burdensomeness and suicidality: Two studies on the suicide notes of those attempting and those completing suicide. Journal of Social and Clinical Psychology. 2002; 21(5): 531-45.

29. Gunn JF, Lester D, Haines J, Williams CL. Thwarted belongingness and perceived burdensomeness in suicide notes. Crisis. 2012; 33(3): 178-81.

30. Lester D, Gunn IJF. Perceived burdensomeness and thwarted belonging: An investigation of the interpersonal theory of suicide. Clinical Neuropsychiatry. 2012; 9(6): 221-4.

31. Fernández-Cabana M, Jiménez-Féliz J, Alves-Pérez MT, Mateos R, Gómez-Reino I, García-Caballero AA. Linguistic analysis of suicide notes. The European Journal of Psychiatry. 2015; 29(2).

32. Ramírez-Esparza N, Pennebaker JW, García FA, Suriá R. La psicología del uso de las palabras: Un programa de computadora que analiza textos en español. Revista Mexicana de Psicología. 2007; 24(1): 85-99.

33. Värnik A, Kõlves K, van der Feltz-Cornelis CM, Marusic A, Oskarsson H, Palmer A, et al. Suicide methods in Europe: a gender-specific analysis of countries participating in the "European Alliance Against Depression". Journal of Epidemiology and Community Health. 2008; 62(6): 545-51.

34. Bertolote JM, Fleischmann A. A global perspective in the epidemiology of suicide. Suicidologi. 2002; 7(2): 6-8.

35. Cavanagh JT, Carson AJ, Sharpe M, Lawrie SM. Psychological autopsy studies of suicide: a systematic review. Psychological Medicine. 2003; 33(3): 395-405.

36. Nock MK, Hwang I, Sampson NA, Kessler RC. Mental disorders, comorbidity and suicidal behavior: results from the National Comorbidity Survey Replication. Molecular Psychiatry. 2010; 15(8): 868-76.
37. Link BG, Yang LH, Phelan JC, Collins PY. Measuring mental illness stigma. Schizophrenia Bulletin. 2004; 30(3): 511-41.

38. Ostman M, Kjellin L. Stigma by association: psychological factors in relatives of people with mental illness. The British Journal of Psychiatry: the Journal of Mental Science. 2002; 181: 494-8.

39. Hawton K. Sex and suicide. Gender differences in suicidal behaviour. The British Journal of Psychiatry: the Journal of Mental Science. 2000; 177: 484-5.

40. Bruffaerts R, Demyttenaere K, Hwang I, Chiu WT, Sampson N, Kessler RC, et al. Treatment of suicidal people around the world. The British Journal of Psychiatry: the Journal of Mental Science. 2011; 199(1): 64-70.

41. Shah A. A replication of the relationship between elderly suicides rates and elderly dependency ratios: a crossnational study. Journal of Injury \& Violence Research. 2010; 2(1): 19-24.

42. Schmidtke A, Bille-Brahe U, DeLeo D, Kerkhof A, Bjerke T, Crepet P, et al. Attempted suicide in Europe: rates, trends and sociodemographic characteristics of suicide attempters during the period 1989-1992. Results of the WHO/EURO Multicentre Study on Parasuicide. Acta Psychiatrica Scandinavica. 1996; 93(5): 327-38.

43. Brown GK, Henriques GR, Sosdjan D, Beck AT. Suicide intent and accurate expectations of lethality: predictors of medical lethality of suicide attempts. Journal of Consulting and Clinical Psychology. 2004; 72(6): 1170-4.

44. Beautrais AL. Suicide and serious suicide attempts in youth: a multiple-group comparison study. The American Journal of Psychiatry. 2003; 160(6): 1093-9.

45. Denning DG, Conwell Y, King D, Cox C. Method choice, intent, and gender in completed suicide. Suicide \& Life-Threatening Behavior. 2000; 30(3): 282-8.

46. Newman ML, Groom CJ, Handelman LD, Pennebaker JP. Gender differences in language use: An analysis of 14,000 text samples. Discourse Process. 2008; 45: 211-36.

47. Wu YW, Su YJ, Chen CK. Clinical characteristics, precipitating stressors, and correlates of lethality among suicide attempters. Chang Gung Medical Journal. 2009; 32(5): 543-52.

48. Berry DS, Hiller WS, Mueller JS, Pennebaker JW. Linguistic bases of social perception. Personality \& Social Psychology Bulletin. 1997; 5: 526-37.

49. Cohn MA, Mehl MR, Pennebaker JW. Linguistic markers of psychological change surrounding September 11, 2001. Psychological Science. 2004; 15(10): 687-93. 
50. Pennebaker JW, Slatcher RB, Chung CK. Linguistic markers of psychological state through media interviews: John Kerry and John Edwards in 2004, Al Gore in 2000. Analyses of Social Issues and Public Policy. 2005; 5: 1-9.

51. Pennebaker JW, Lay T. Language use and personality during crises: Analyses of Mayor Rudolph Giuliani's press conferences. Journal of Research in Personality. 2002; 36: 271-82.

52. Haensch G. Español de América y español de Europa ( $2^{a}$ parte). Panacea Boletín de Medicina y Traducción. 2002; 3(7): 37-64.
* Corresponding author: M. Fernández-Cabana Department of Psychiatry School of Medicine

University of Santiago de Compostela Praza do Obradoiro

Santiago de Compostela 15782, Spain Phone number: 34-981-950901

E-mail: mercedes.fcabana@gmail.com 